\title{
Capital social, confiance et développement territorial. Une étude appliquée en Kabylie
}

Social capital, trustworthiness and territorial development. An applied study in Kabylia

\section{Cécile Perret et Belaïd Abrika}

\section{OpenEdition}

Journals

Édition électronique

URL : http://journals.openedition.org/developpementdurable/11340

DOI : 10.4000/developpementdurable. 11340

ISSN : $1772-9971$

Éditeur

Association DD\&T

Référence électronique

Cécile Perret et Belaïd Abrika, « Capital social, confiance et développement territorial. Une étude appliquée en Kabylie », Développement durable et territoires [En ligne], Vol. 7, n² I Juillet 2016, mis en ligne le 28 juillet 2016, consulté le 19 avril 2019. URL : http://journals.openedition.org/ developpementdurable/11340; DOI : 10.4000/developpementdurable.11340

Ce document a été généré automatiquement le 19 avril 2019.

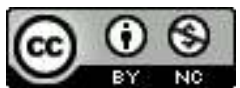

Développement Durable et Territoires est mis à disposition selon les termes de la licence Creative Commons Attribution - Pas d'Utilisation Commerciale 4.0 International. 


\title{
Capital social, confiance et développement territorial. Une étude appliquée en Kabylie
}

Social capital, trustworthiness and territorial development. An applied study in Kabylia

\author{
Cécile Perret et Belaïd Abrika
}

1 Dans un essai intitulé La société de défiance. Comment le modèle social français s'autodétruit (2007), Yann Algan et Pierre Cahuc soulignent que les effets pervers du manque de confiance affectent principalement l'efficacité du marché, la qualité du dialogue social et le civisme. Dans les sociétés de tradition orale, la notion de confiance prend une acuité particulière car elle conditionne certaines transactions réalisées sans contrat formel.

La socialisation prédispose à obéir à un certain nombre de normes qu'elles soient morales, sociales ou personnelles. "Assez souvent, la disposition à être honnête [ou confiant] sera envers les membres de certains groupes particuliers (clans, voisins ou groupe ethnique), et non d'autres » (Dasgupta, 2012, p. 55). Cet état de fait est particulièrement intéressant à étudier dans des pays composés de subcultures différentes comme c'est le cas en Algérie. Ces subcultures, qui ont bien évidemment un fond culturel commun, se différencient essentiellement par leur capital social civil (organisations particulières émanant de la société civile). Dans cet article, nous nous concentrons sur la subculture des Monts de Kabylie dont le taux d'homogénéité de la population est élevé ${ }^{1}$ et qui a conservé un système de gouvernance traditionnel des villages ainsi qu'une intensité des liens horizontaux. En 1958, Bourdieu écrivait à ce sujet : à "l'imperfection des techniques répond une perfection hyperbolique du lien social, comme si la précarité de l'ajustement à l'environnement naturel trouvait contrepoids dans l'excellence de l'ajustement social [...] $»^{2}$. Aujourd'hui, dans ce territoire vécu (Perret et Gagnon, 2016), la "capacité à créer du lien » est toujours exacerbée bien qu'elle soit malmenée par l'évolution de la société (décennie noire, système redistributif qui s'améliore et amoindrit la nécessité des solidarités traditionnelles, etc.) 
3 Les liens qui unissent les acteurs d'un territoire ${ }^{3}$ peuvent être examinés à la lumière du concept de capital social qui s'inscrit dans les relations intragroupe, intergroupe et environnementale. Pour reprendre la terminologie de Bernard Pecqueur (2003), la question est finalement de savoir si cette " capacité à créer du lien » est une ressource spécifique transformable en actif spécifique. Autrement dit, le lien social peut-il engendrer un capital social?

4 Dasgupta $(2010,47)$ soutient que le développement de la confiance entre les Hommes, clef de la coopération, est la condition au progrès économique. Il retient une définition restrictive du capital social comme des "réseaux entre les personnes dont les membres développent et maintiennent la confiance entre eux pour tenir leurs promesses à travers l'application mutuelle des accords " et montre que, s'il est convenablement dirigé, le capital social peut permettre le maintien de la confiance (Dasgupta, 2010, p. 50). A contrario, s'il est mal dirigé, il peut entraver le développement voire faire régresser l'économie.

Ceci une fois posé, notre questionnement est le suivant: la «valeur confiance " (sousentendu avoir un comportement digne de confiance) est-elle toujours aussi importante, sur quoi repose-telle et a-t-elle un impact sur le développement en Kabylie ? Afin d'y répondre, cet article présente dans une première partie le concept polysémique de capital social (Bourdieu, 1980...) dont nous distinguons les différentes dimensions. Une discussion sur la nature du lien social nous permet de compléter l'analyse en caractérisant la qualité des liens dans la structure sociopolitique des villages kabyles. Dans la seconde partie, nous précisons ce que recouvre le terme de confiance et les éléments qui la sous-tendent avant d'analyser le lien entre la confiance et le développement (Putnam, 1993 ; Algan et Cahuc, 2007). Les travaux de Fukuyama (1995) et de Dasgupta (2010) nous amènent à souligner l'encastrement culturel de la confiance. Enfin, notre troisième partie présente les résultats d'une enquête exploratoire qualitative sur la confiance qui nous permet, en particulier, de conclure au manque de confiance dans les institutions et à l'absence d'un renfermement communautaire.

\section{Capital social et lien social}

\subsection{Les principales conceptions du capital social}

Bourdieu (1980) a développé une vision instrumentale du capital social qu'il considère en tant qu'ensemble de ressources actuelles ou potentielles qui sont liées à la possession d'un réseau durable de relations. Les individus investissent stratégiquement en capital social dans la perspective d'en retirer un bénéfice. C'est également un héritage qui constitue "un attribut du groupe ou du réseau social auquel appartient l'individu» (Kamanzi et al., 2007, p. 129). Il peut devenir un instrument de domination, étant à la fois l'effet et la cause de l'institution sociale et des inégalités de classes dans une société traversée de rapports sociaux (Barret et Solo Maciel, 2004). Coleman (1988) fait, lui, référence aux obligations, aux responsabilités, aux attentes, aux normes et aux relations de confiance dont un individu jouit auprès des autres personnes d'un réseau auquel il adhère de façon volontaire. Le capital social a deux fonctions principales : il permet de faire circuler l'information, et ainsi d'accéder à des opportunités, et il permet un contrôle des actions individuelles (par exemple échanger sans contrat formel au sein d'une communauté particulière). Coleman souligne également que les ressources privées qu'un individu retire de la valorisation de son capital social ont des répercussions au plan 
macroéconomique. Enfin, Putnam $(1995,2000)$ recentre l'analyse sur la qualité des relations et la confiance. Le capital social favorise l'action collective et la coopération : au sein d'un groupe « irrigué » par des réseaux de relations interpersonnelles, des attitudes de confiance générale entre les membres et des normes de réciprocité qui facilitent l'action collective vont se développer (Houard et Jacquemain, 2006, p. 8).

7 Le terme de capital est cependant sujet à controverse car il suppose la durée dans le temps, des sacrifices délibérés au présent pour des bénéfices futurs et l'aliénabilité (Arrow, 1999, p. 4). Or, les relations sociales peuvent être construites pour des raisons non économiques (Arrow, 1999, p. 3). Solow va dans le même sens en écrivant que le capital social est " un ensemble d'éléments comme la confiance, la volonté et la capacité à coopérer et se coordonner, l'habitude de contribuer à l'effort commun même si personne ne regarde» (Solow, 1999, p. 7). Dasgupta (2010) souligne également que c'est le développement de la confiance entre les personnes qui conditionne le développement économique. Le capital social, défini ici comme des « réseaux entre les personnes ", permet d'établir et maintenir la confiance. Mais, s'il opère dans un mauvais milieu (mafieux par exemple), il peut au contraire entraver le développement économique. Ainsi, des réseaux denses n'améliorent pas forcément la situation économique globale, du moins pas dans le long terme (Gambetta, 1993 in Dasgupta, 2010, p. 49).

8 La définition de Sirven (2003) nous intéresse particulièrement car elle met en évidence le fait que le capital social est un ensemble de ressources accessibles grâce à des réseaux de relations qui peuvent s'établir au sein d'entités formelles ou informelles et qui vont engendrer des obligations reposant sur des règles, des normes et des traditions de réciprocité. Le lien social peut devenir un capital s'il est organisé (structuré en réseau) et valorisé. La fréquence des interactions et leur qualité peut engendrer des relations de confiance et des comportements vertueux.

\subsection{Lien social fermé et lien social ouvert}

9 Les liens dits «fermés », bonding ties selon la terminologie de la Banque Mondiale (2000), sont des liens horizontaux de type communautaire qui peuvent être le signe d'une cohésion sociale s'ils sont intenses et harmonieux tandis que les liens dits « ouverts » sont ceux que l'on trouve entre deux collègues, des amis éloignés, etc. En Kabylie, les liens de type bonding sont primordiaux car «la place d'unité sociale et politique qu'occupe la famille se justifie par une vision profonde de la vie en société» (Adli, 2010-a, p. 20). Les membres de la communauté villageoise sont, en outre, animés par l'esprit du «vivre ensemble » en respectant les normes morales et sociales communautaires. Un dicton précise d'ailleurs "Akhdhem aken khedmen meden" que l'on pourrait traduire par «ne déroge pas aux normes du village ». Pourquoi dans certaines sociétés ces liens sont plus forts que dans d'autres? L'histoire, la culture des populations, les conditionnements socioéconomiques et politiques déterminent «l'intensité » des réseaux (Bucolo, 2004, p. 71).

10 Les liens de type linking caractérisent des interactions entre des agents de statuts différents (entre personnes de deux ethnies différentes par exemple). Ces liens verticaux se caractérisent par des transactions de réciprocité qui obligent à la poursuite des échanges. Leur fréquence élevée peut amener à une convergence des représentations (Angeon, Caron et Lardon, 2006, p. 10). Ces liens déterminent le degré d'ouverture/ 
insertion de la communauté dans un ensemble plus vaste. Ce sont ces liens qui font le ciment d'une communauté nationale composée de subcultures.

11 Enfin, les liens de type bridging lient des agents distants géographiquement ou bien le lien n'est pas activé en permanence (Angeon, Caron et Lardon, 2006, p. 13). Les liens entre les villageois résidents et les émigrés du village pourraient être caractérisés de cette façon, bien que l'on sache qu'il existe une intensité réelle des communications entre les deux (appels téléphoniques réguliers, voyages au pays, etc.). Ces liens sont essentiels au développement des villages kabyles car ils permettent à la fois circulation des techniques, des idées et d'abonder à la caisse ${ }^{4}$ des villages qui permet la mise en œuvre de projets (construction d'un dispensaire, d'une fontaine, etc.) grâce au travail collectif et bénévole des villageois (Perret et Paranque, 2014).

Les trois types de liens (bonding, linking et bridging) dessinent le maillage au sein de la communauté et son insertion/articulation dans/avec d'autres groupes. Cependant, un renforcement des liens de type bonding au détriment des autres peut signifier le repli sur soi, voire peut être un danger pour la cohésion du pays (Colletta et Cullen, 2000). Woolcock (1998) qualifie cette situation (intégration interne exagérée et manque de liaisons externes) d' « amoralisme familier » (in Martens, 2003, p. 117). Les normes sociales sont alors déterminées par la communauté qui promeut l'unité interne et décourage voire punit le contact ou la solidarité avec les membres extérieurs à la communauté. En sus, le renfermement dans des réseaux familiaux ou ethniques limite les ressources en capital social disponibles et, nuisant au développement des relations « ouvertes ", peut avoir des effets négatifs sur le développement (Bucolo 2004, p. 71). Tout le problème est alors de savoir à partir de quel seuil les liens affectifs peuvent devenir un obstacle.

\subsection{L'intensité des liens horizontaux dans l'organisation traditionnelle de la société kabyle}

13 Comme bon nombre d'économistes et d'anthropologues l'ont souligné, des organisations informelles sont créées en l'absence d'institutions formelles fortes (Woolcock, 2001, p.13). En Kabylie, ce sont les comités de village ${ }^{5}$, survivance de la forme d'organisation traditionnelle berbère la plus répandue chez les sédentaires. "Sorte de république villageoise $»^{6}$ masculine, elle comporte une assemblée du village (taajmat), organe délibérant, législatif et exécutif. Le chef du village est élu par les membres de tajmaat composée des représentants des familles du village, les taman. Les taman veillent au respect du règlement intérieur ${ }^{7}$ du village (lqanoun taddart), à la bonne gestion des projets d'utilité collective (répartition du travail collectif en particulier) et aux conflits internes au village (Perret et Abrika, 2014). Comme l'écrivait Bourdieu (1958), l'organisation traditionnelle pourrait être représentée par une série de cercles concentriques partant de la famille pour aller vers la confédération de tribus, les liens de solidarité et leur fréquence étant d'autant plus relâchés que l'on s'écarte du centre (cf. figure. 1 cidessous). 
Figure 1. La structure sociopolitique traditionnelle kabyle

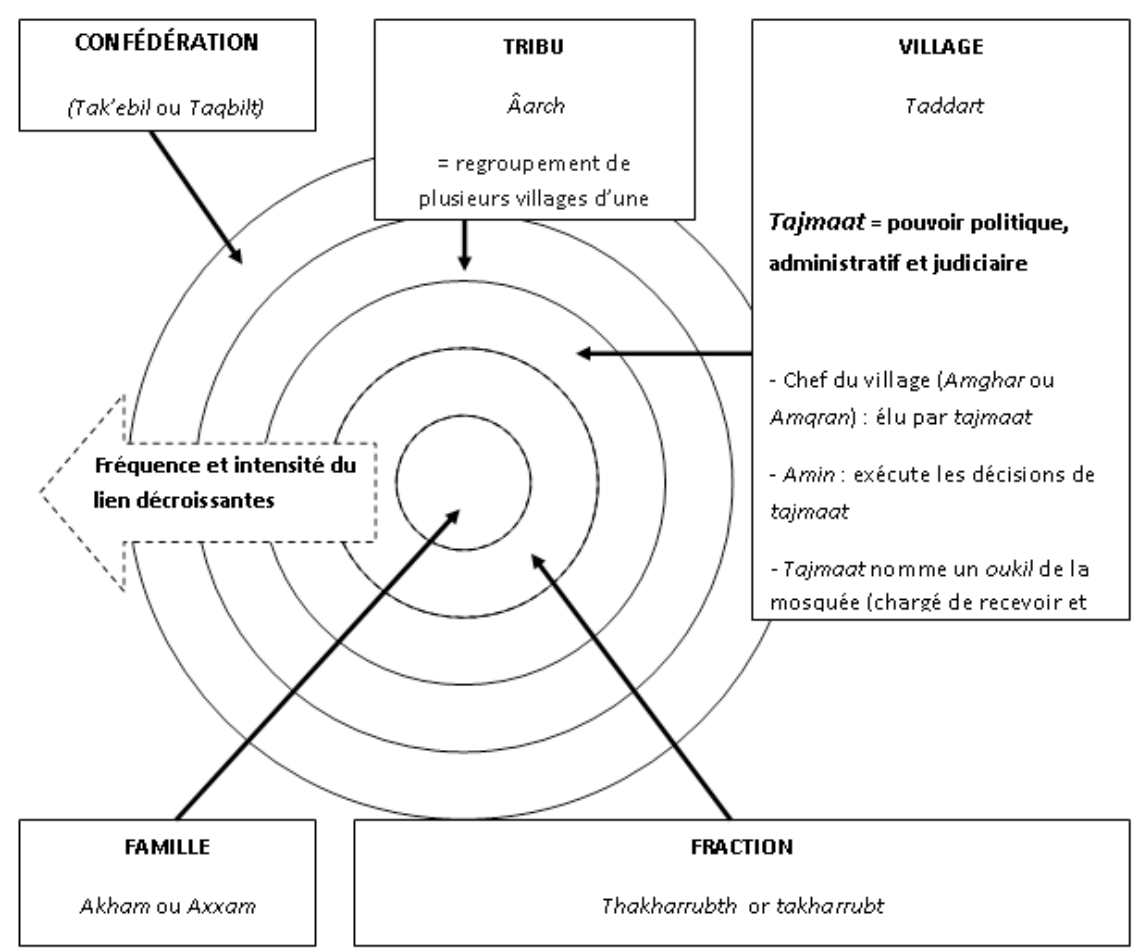

Source : réalisation personnelle Perret et Abrika.

La Kabylie est caractérisée par sa capacité à créer des çof, une association d'assistance mutuelle ponctuelle. Son but est bien défini par le vieil adage » ouinek aïnoun ith idhelem ner medhloum ", "aide les tiens, qu'ils aient tort ou raison " (Hanoteau et Letourneux, 2003, Tome deuxième, 14). La qualité des liens créés par les çof est d'autant plus importante qu'il s'agit des çof d'un même village ou d'une même tribu. Cheikh Mohand ${ }^{8}$ identifiait les fondements de la société kabyle comme étant tajmaat, la valeur de l'effort et l'importance de la fratrie (Adli, 2010-a, p. 47-48). Les liens de type bonding sous-tendent donc toute l'organisation sociopolitique kabyle.

Il est reconnu que l'organisation des villages kabyles a non seulement permis de pallier les défaillances de l'État (notamment dans les années 90) et financer des projets locaux de développement (Iguersafène, un village de 4500 habitants, a ainsi récemment mis en place un système de tri sélectif et de recyclage des déchets grâce aux moyens et au travail collectif du village) ${ }^{9}$ mais a aussi « contribué à maintenir le lien social malmené par plus d'une décennie de guerre civile et de crise économique " (Direche-Slimani, 2006, p. 183). La "capacité à créer du lien", ressource spécifique car directement issue de la dynamique territoriale (Pecqueur, 2003), se transforme en capital spécifique au service du développement des villages grâce à tajmaat. 


\section{La confiance, une valeur encastrée comme support au développement local}

\subsection{Retour sur la notion de confiance}

La difficulté à bien cerner le terme de confiance est qu'il recouvre différentes acceptions : la confiance de base (familiarity) qui est un état plus ou moins inconscient (cela a toujours été ainsi), la confiance au sens large (confidence) qui se manifeste sur un arrière-fond de confiance de base (on n'est pas sûr de l'issue mais on s'attend à un résultat positif) et la confiance au sens vrai du terme (goodwill trust) qui commence où la calculabilité et le contrôle s'arrêtent (Schuller, 2004, p. 9).

Selon Schuller (2004, p. 15), "la question de la confiance se pose différemment si l'on part de l'hypothèse que l'homme est plutôt égoiste, rationnel ou de l'hypothèse admettant qu'il est un être guidé par des considérations morales dans un système de normes et de conventions». Une distinction pourrait alors être faite en une confiance de nature instrumentale ( j'ai besoin de l'autre ») et une confiance de nature éthique («je me préoccupe de l'autre »). En Kabylie, la notion de parole donnée est primordiale. Traditionnellement, «c'est par le dit et ses métaphores, la loyauté, la bravoure, la science et la connaissance et la valeur du serment et de la parole donnée que l'homme peut prétendre au qualificatif de riche» Adli (2010-a, p. 18). Ce sont ces valeurs immatérielles (tirugza) qui définissent l'argaz ${ }^{10}$ (tabargazt au féminin), terme que l'on pourrait traduire par homme « de parole », courageux ou honnête. Cette valeur (tirurgza) est fondamentale au bon fonctionnement de la société traditionnelle kabyle puisqu'un argaz participe aux travaux collectifs du village, tient sa parole, etc. et donc participe à la cohésion et au développement du village.

La confiance peut être étudiée à plusieurs niveaux. Au sein de la famille, elle réside dans le fait que chacun est concerné par le bien-être de l'autre (éthique, normes...). Dans des relations de proximité, c'est la réputation qui permet son maintien. Enfin, dans le cadre de relations impersonnelles, elle est essentiellement maintenue par des règles de droit. Dans ce dernier cas, la confiance est déplacée au niveau de celui qui détient le pouvoir de coercition, au niveau des institutions. Pour Dasgupta (2010), dans le cas où l'institution (l'État) n'a pas été jugée digne de confiance par le passé, il devient difficile et long de renverser les croyances. Les échanges vont alors se développer au niveau personnel. En Algérie, l'Indice de Perception de la Corruption de Transparency International ${ }^{11}$ est faible ${ }^{12}$. Cette faiblesse pourrait expliquer, en partie, le recours à des liens communautaires et la participation des villageois kabyles à tajmaat.

19 La confiance peut se construire selon diverses modalités (familiarité, habitude, calcul et culture) et compter divers degrés (on peut avoir totalement confiance ou pas du tout). Laurent (2012) distingue deux cas de "vraie confiance » (entre les personnes et envers les institutions), deux cas de "fausse confiance» (la confiance en l'avenir qui est de l'optimisme et la confiance en soi qui est de l'estime de soi) et une "vraie-fausse » confiance (la confiance généralisée).

20 Dans quels environnements sociaux la confiance peut-elle se développer? Selon Dasgupta (2010, p. 53-54), au moins dans les cas d'affection mutuelle et dans le cas de disposition pro-sociale, par exemple une culture commune qui modèle les préférences, les attentes et les comportements. 


\subsection{La confiance et le développement}

21 comme un élément discriminant du développement. Putnam, dans Making democracy work publié en 1993, remarque que les gouvernements ${ }^{13} \mathrm{du}$ Nord de l'Italie ont mieux réussi économiquement que ceux du Sud de l'Italie. Pour expliquer cette différence, il se concentre sur la «communauté civique» dans laquelle baigne chacun de ces gouvernements régionaux. Les indicateurs retenus par Putnam pour évaluer le civisme de la vie régionale sont la qualité de la vie associative, la lecture de la presse et la participation aux référendums et aux votes. En Kabylie, plus que le nombre d'associations, c'est certainement la persistance et l'implication des villageois dans les comités de village qu'il conviendrait de prendre en compte. D'autre part, en Kabylie, l'abstention aux élections est, contrairement à ce que l'intuition pourrait laisser croire, souvent le signe d'un engagement fort dans la communauté de ce territoire qui signale par ce comportement la méfiance qu'elle éprouve envers les institutions de l'État (rejet du « système », peur d'élections « truquées », etc.).

Dans leur essai, Algan et Cahuc (2007) soutiennent qu'en France le déficit de confiance constitue un facteur de vulnérabilité de l'économie française. Ils soulignent que le mode de fonctionnement de la Sécurité sociale française - basé sur un fonctionnement corporatiste qui octroie les droits sociaux en fonction du statut et de la profession de chacun - et la centralisation hiérarchique des décisions par l'État segmentent la société en institutionnalisant les différences entre citoyens. Ceci, en opacifiant les relations sociales, engendre la défiance ${ }^{14}$ des citoyens et l'idée que pour réussir il faut contourner les règles.

En Algérie, pays à l'État fortement centralisé, il est fréquent d'entendre des citoyens critiquer « le système » (qui recouvre pour eux à la fois les politiques, les dirigeants de grandes entreprises nationales, les institutions etc.) selon eux fondamentalement corrompu et contrôlé par une "mafia politico-militaire». Pour autant, une pratique courante consiste à faire appel à ses connaissances personnelles (tamusni ou timusniwin au pluriel) pour débloquer certaines situations (faire avancer un dossier d'attribution de logement, trouver un travail, etc.) Cette "connaissance" peut d'ailleurs parfois se rapprocher du « dark side » puisqu'elle est aussi utilisée pour obtenir des passe-droits. La Banque Mondiale souligne elle-même que ce type de recours peut conduire à la discrimination ou à la corruption ("[...] personal connections can be used to unfairly discriminate, distort and corrupt" (Woolcock and Narayan, 2000, p. 226).

\subsection{Confiance et culture}

L'attitude de confiance envers autrui a-t-elle des caractéristiques sociales et culturelles particulières? Dans Trust (publié en 1995), Francis Fukuyama explique qu'au sein de structures sociales, la confiance réciproque entre les Hommes (la confiance est, pour lui, liée aux traditions religieuses et culturelles), le capital social et la réussite économique capitaliste vont de pair et conditionnent l'avènement de la démocratie et le bonheur collectif (Naves, 2003, p. 477). Fukuyama souligne également le rôle de la dimension culturelle et $\mathrm{du}$ fonctionnement harmonieux de la société civile dans la réussite économique et la solidité politique. Une société où l'individualisme finit par primer sur la 
solidarité pourrait alors décliner. Une société de confiance fortement pourvue en capital social serait, elle, capable de s'auto-organiser pour satisfaire des objectifs politiques et économiques communs. En résumé, selon Fukuyama, et en dehors de toute considération sur le rôle de l'État, certaines traditions (religion, solidarités communautaire, etc.) seraient à l'origine de la bonne ou de la mauvaise santé du capital social et de la confiance d'une société (Naves, 2003, p. 481). Le rôle de la solidarité familiale, que Fukuyama souligne (certaines communautés familiales seraient plus propices que d'autres au développement économique), est très intéressant dans le cas de la Kabylie qui nous concerne ici. En effet, la famille a un rôle prépondérant dans la création d'entreprises et le financement des petites entreprises est la plupart du temps familial. La communauté d'appartenance dans un sens plus large (village, ville, wilaya) peut également avoir son importance. Ainsi, la fierté d'appartenir à un territoire qui se développe peut parfois expliquer une certaine entraide (échanges sur les techniques par exemple) entre les artisans d'une même région (Perret, 2012, p. 62).

\section{La confiance en Kabylie, une approche empirique}

Afin d'avoir une idée plus fine des différentes formes de confiance ou de l'optimisme (ou du pessimisme) ambiant en Kabylie, nous avons élaboré un questionnaire ${ }^{15}$ inspiré de celui du World Values Survey ${ }^{16}$. Nous avons notamment posé des questions sur les valeurs auxquelles les répondants sont attachés, sur leur niveau de confiance envers les personnes ou les institutions, sur la perception de la corruption, sur leur optimisme ou pessimisme et enfin quelques questions nous permettant d'analyser un éventuel enfermement communautaire qui pourrait nuire à la création d'entreprises et à la dynamique territoriale. Le questionnaire a été administré en ligne à des étudiants de l'université de Tizi-Ouzou de façon à préserver leur anonymat sur un sujet sensible. Il a également circulé en dehors de l'Université grâce à une mise en ligne sur le site internet d'un quotidien national algérien en langue française ${ }^{17}$ beaucoup lu en Kabylie. Dans cet article, nous avons retenu 182 personnes dont les questionnaires sont suffisamment documentés et dont les parents vivent dans la wilaya de Tizi-Ouzou. Le nombre d'enquêtés étant très réduit par rapport à la population totale (1 119646 habitants dans la Wilaya de Tizi Ouzou, 694749 habitants dans la Wilaya de Bouira, une diaspora...) il ne peut être question ici d'une enquête statistique avec toutes les opérations quantitatives qui s'y rattachent. Pour les questions à échelle, nous avons calculé une fréquence et une moyenne, les questions ouvertes nous ont aidés à interpréter les résultats.

\footnotetext{
Sur ces 182 personnes (dont 118 hommes), 84 sont des étudiants, 35 sont cadres ou ont une profession intellectuelle supérieure, 18 sont artisans, commerçants ou chefs d'entreprise, 17 sont employés, 14 sont sans emploi, 10 sont de professions intermédiaires, 2 sont des ouvriers et, enfin, 2 n'ont pas répondu. Cet échantillon comporte donc des personnes de niveau socioculturel voire économique plutôt élevé (tous les Algériens ne disposent pas d'un ordinateur), ce qui peut représenter un biais.
}

Quelles sont les valeurs auxquelles ces 182 individus attachent de l'importance ? Lorsque nous leur avons demandé de choisir dans une liste les 3 valeurs auxquelles ils accordent le plus d'importance, la confiance est la valeur la plus fréquemment citée (127 citations soit 
$69,8 \%$ des répondants), suivie de l'amour (99 citations soit 54,4\% des répondants) et de l'entraide/la solidarité (88 citations soit $48,4 \%$ des répondants) (Tableau 2). Un quelconque impact de l'âge des répondants sur ces choix n'est pas significatif au seuil de $5 \%$. Par contre, si l'on analyse l'impact du genre sur les réponses, pour les hommes la démocratie et la laïcité sont surreprésentées et pour les femmes c'est la piété/la foi.

Tableau 1. Parmi les valeurs suivantes, choisissez en 3 auxquelles vous attachez le plus d'importance

\begin{tabular}{|l|l|l|}
\hline & Nombre de citations & \% du nombre d'observations \\
\hline La confiance (tirugza) & 127 & $69,8 \%$ \\
\hline L'amour & 99 & $54,4 \%$ \\
\hline L'entraide/la solidarité & 88 & $48,4 \%$ \\
\hline La démocratie & 72 & $39,6 \%$ \\
\hline L'amitié & 53 & $29,1 \%$ \\
\hline La laïcité & 52 & $28,6 \%$ \\
\hline La piété/la foi & 33 & $18,1 \%$ \\
\hline La charité & 14 & $7,7 \%$ \\
\hline Non réponse & 1 & $0,5 \%$ \\
\hline
\end{tabular}

Les personnes interrogées déclarent également qu'elles attachent beaucoup d'importance à la formation (moyenne de 4,72/5) et aux amis (moyenne de 3,99/5 (Tableau 3)). Si la religion arrive en dernière position dans le classement (le score est moyen $(2,41 / 5)), 117$ personnes se déclarent musulmanes, 13 athées, 2 chrétiennes et 63 laïques (plusieurs réponses étaient admises pour cette question).

Tableau 2. Indiquez pour chacune des propositions suivantes si cela à de l'importance pour vous

\begin{tabular}{|l|l|l|}
\hline & Moyenne & Écart-type \\
\hline Formation & 4,72 & 0,59 \\
\hline Amis & 3,99 & 0,91 \\
\hline Travail & 3,71 & 0,76 \\
\hline Famille & 3,68 & 0,8 \\
\hline Loisirs & 3,37 & 0,78 \\
\hline Bénévolat/monde & 3,18 & 0,91 \\
\hline associatif & & \\
\hline
\end{tabular}




\begin{tabular}{|l|l|l|}
\hline Politique & 2,46 & 1,12 \\
\hline Religion & 2,41 & 1,26 \\
\hline $\begin{array}{l}\text { Valorisation des échelons }: 1=\text { sans importance } ; 2=\text { peu important } ; 3=\text { assez important } ; 4= \\
\text { important ; } 5 \text { = très important. Réponses effectives }=180 . \text { Taux de réponse }=98,9 \% .\end{array}$ \\
\hline
\end{tabular}

Les réponses concernant la confiance dans les personnes nous montrent un véritable déficit de confiance envers les hommes politiques puisque sur une échelle allant de " pas du tout confiance » $(=1)$ à " totalement confiance » $(=5)$, la moyenne est de $1,27 / 5$ pour les hommes politiques au niveau central et 1,44/5 pour les hommes politiques au niveau local (Tableau 4). Ce sont logiquement les personnes appartenant à nos groupes primaires en qui l'on fait confiance (moyenne de 4,62/ 5 pour la famille). Concernant les comités de village ou comités de quartiers (version urbaine des comités de village), une distinction entre les deux nous aurait peut-être permis de différencier les moyennes. On peut penser que dans les zones rurales de montagne la moyenne aurait été supérieure à 2,45/5 car tajmaat est l'émanation directe de la communauté villageoise où tout le monde se connait. Ou bien cela est-il tout simplement le signe que dans certains villages tajmaat ne fonctionne plus vraiment de façon consensuelle.

Tableau 3. En qui faites-vous confiance ? (de « Pas du tout confiance à « Totalement confiance »)

\begin{tabular}{|c|c|c|c|c|c|}
\hline & $\begin{array}{l}\text { Moyenne } \\
/ 5\end{array}$ & Écart-type> & Min. & Max. & $\begin{array}{l}\text { Taux de } \\
\text { réponse }\end{array}$ \\
\hline $\begin{array}{l}\text { Les hommes politiques } \\
\text { au niveau central }\end{array}$ & 1,27 & 0,58 & 1 & 4 & $98,9 \%$ \\
\hline $\begin{array}{l}\text { Les hommes politiques } \\
\text { au niveau local }\end{array}$ & 1,44 & 0,70 & 1 & 4 & $98,9 \%$ \\
\hline $\begin{array}{l}\text { Les membres de mon } \\
\text { comité de village ou } \\
\text { de quartier }\end{array}$ & 2,34 & 1,13 & 1 & 5 & $98,9 \%$ \\
\hline $\begin{array}{l}\text { Les habitants de mon } \\
\text { quartier/mon village }\end{array}$ & 2,45 & 0,98 & 1 & 5 & $100 \%$ \\
\hline $\begin{array}{l}\text { Mes collègues de } \\
\text { travail }\end{array}$ & 2,55 & 0,95 & 1 & 5 & $95,1 \%$ \\
\hline Mes connaissances & 2,90 & 1,11 & 1 & 5 & $98,4 \%$ \\
\hline Mes amis & 3,41 & 1,01 & 1 & 5 & $97,3 \%$ \\
\hline Ma famille & 4,62 & 0,8 & 1 & 5 & $99,5 \%$ \\
\hline \multicolumn{6}{|c|}{ Valorisation des échelons: $1=$ pas du tout confiance ; $2=$ plutôt pas confiance ; $3=$} \\
\hline
\end{tabular}


Concernant les institutions, le déficit de confiance est criant. L'État central (moyenne de $1,53 / 5$ ) et la Wilaya (moyenne de 1,59/5) obtiennent un niveau de confiance moyen très faible (Tableau 5). Les personnes interrogées accordent plus de confiance aux institutions relevant de la tradition (comités de village ou de quartier (moyenne de 2,49/5)) et aux

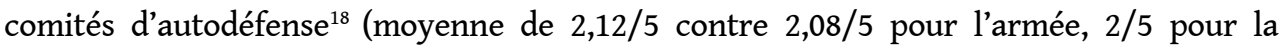
police et $1,97 / 5$ pour la gendarmerie) qu'aux institutions relevant de l'État.

Tableau 4. En quelles institutions faites-vous confiance ? (de « Pas du tout confiance » à " Totalement confiance")

\begin{tabular}{|c|c|c|c|c|c|}
\hline & $\begin{array}{l}\text { Moyenne } \\
/ 5\end{array}$ & Écart-type & Min & Max & $\begin{array}{l}\text { Taux } \\
\text { réponse }\end{array}$ \\
\hline L'État central & 1,53 & 0,93 & 1 & 5 & $97,3 \%$ \\
\hline La Wilaya & 1,59 & 0,89 & 1 & 5 & $97,8 \%$ \\
\hline $\begin{array}{l}\text { Le système } \\
\text { judiciaire algérien }\end{array}$ & 1,64 & 1,01 & 1 & 5 & $98,4 \%$ \\
\hline $\begin{array}{l}\text { L'assemblée } \\
\text { populaire } \\
\text { communale (APC) }\end{array}$ & 1,74 & 0,89 & 1 & 5 & $98,9 \%$ \\
\hline $\begin{array}{l}\text { Le système éducatif } \\
\text { algérien }\end{array}$ & 1,77 & 1,06 & 1 & 5 & $99,5 \%$ \\
\hline La gendarmerie & 1,97 & 1,22 & 1 & 5 & $99,5 \%$ \\
\hline La police & 2,00 & 1,23 & 1 & 5 & $97,3 \%$ \\
\hline $\begin{array}{l}\text { Le système de } \\
\text { protection sociale } \\
\text { algérien }\end{array}$ & 2,01 & 1,04 & 1 & 5 & $98,9 \%$ \\
\hline L'armée & 2,08 & 1,29 & 1 & 5 & $97,3 \%$ \\
\hline $\begin{array}{l}\text { Les comités } \\
\text { d'autodéfense }\end{array}$ & 2,12 & 1,19 & 1 & 5 & $98,9 \%$ \\
\hline $\begin{array}{l}\text { Les comités de } \\
\text { village ou de } \\
\text { quartier }\end{array}$ & 2,49 & 1,20 & 1 & 5 & $98,9 \%$ \\
\hline
\end{tabular}

Ce déficit de confiance dans les institutions est certainement à relier, en partie, à la perception de la corruption. Le niveau de perception de la corruption est particulièrement fort dans les institutions de proximité (moyenne de 3,06/5 pour la Wilaya et 2,84/5 pour l'Assemblée Populaire Communale (Tableau 6)). 
Tableau 5. Comment percevez-vous l'état de la corruption dans votre pays aux différents niveaux suivants ? (de " pas du tout corrompu » (=1) à « totalement corrompu » (=5))

\begin{tabular}{|l|l|l|l|l|l|}
\hline & Moyenne/5 & Écart-type & Min & Max & $\begin{array}{l}\text { Taux } \\
\text { réponse }\end{array}$ \\
\hline Wilaya & 3,06 & 1,13 & 1 & 4 & $92,2 \%$ \\
\hline APC & 2,84 & 0,98 & 1 & 4 & $94,9 \%$ \\
\hline État central & 2,77 & 1,27 & 1 & 4 & $90,3 \%$ \\
\hline $\begin{array}{l}\text { Comités } \\
\text { village/ } \\
\text { quartier }\end{array}$ & 1,94 & 0,90 & 1 & 4 & $97,6 \%$ \\
\hline $\begin{array}{l}\text { Valorisation des échelons }: 1=\text { pas du tout corrompu ; } 2=\text { un peu corrompu ; } 3=\text { moyennement } \\
\text { corrompu ; }=\text { très corrompu ; } 5=\text { totalement corrompu. }\end{array}$ \\
\hline
\end{tabular}

$70,9 \%$ des 182 personnes interrogées (avec un taux de réponse de 98,4\%) déclarent faire plus facilement confiance aux membres de leur famille qu'à des personnes extérieures au cercle familial. L'étude des verbatim est intéressante, le thème des liens du sang ressortant 9 fois ("à cause des liens du sang qui nous unissent», "on partage le même sang, le même honneur, la même mentalité ", "les liens du sang ne trompent pas et ne trahissent pas non plus ", etc.). Le thème de la trahison est également évoqué : «ils ne vont pas trahir ma confiance ». La confiance se développe ici dans le cas de l'affection mutuelle dont parle Dasgupta (2010, p. 53-54). 53,7\% des 182 répondants déclarent faire plus facilement confiance aux personnes vivant dans la wilaya de Tizi-Ouzou qu'aux personnes vivant en dehors de cette wilaya (avec un taux de réponse de 97,3\%). Concernant ces personnes, le thème de la «kabylité » et de la proximité des valeurs est évoqué: «[...]on a la même culture et les mêmes traditions kabyles ", "vue mon origine kabyle [...], j'ai peur d'être trahi par ceux qui ne le sont pas ", "vue mon origine kabyle je préfere faire confiance aux gens de ma wilaya ", " on partage un passé commun et un avenir commun ", " on partage la même façon de voir les choses ", "la proximité des valeurs", "la même culture ", etc. Il s'agit là de disposition pro-sociale (Dasguta, 2010, p. 53-54). Les personnes qui, a contrario, déclarent qu'elles ne font pas plus confiance aux personnes résidant dans leur wilaya qu'aux autres développent des arguments du type : "on est tous des algériens et frères ", " que la personne soit en dehors de la wilaya ou en dehors du pays, la confiance reste une confiance, l'amitié n'a pas de frontières ", "le critère de la wilaya n'a pas de sens en l'occurrence ", etc.

Nous leur avons également demandé si, dans le cadre d'une création personnelle d'entreprise, ils prendraient en priorité un associé membre du cercle familial. À cette question, 66,9 \% répondent qu'ils prendraient plutôt un membre de leur famille (avec un taux de réponse de $97,8 \%$ ). Ce résultat est sans doute à relier au mode de financement familial des entreprises. Nous avons à nouveau posé cette question mais par rapport à une personne de leur wilaya de résidence. Dans ce dernier cas, 59,4\% indiquent qu'il leur est indifférent que leur associé ne soit pas de la même wilaya de résidence. Nous leur laissions également la possibilité d'expliquer leur choix grâce à une question ouverte. Sur les personnes qui ont explicité ce choix, il ressort des réponses obtenues que c'est la 
compétence, et non l'origine de la personne, qui compte. Si l'on préfère quelqu'un de sa wilaya en cas de création d'entreprise c'est aussi pour des raisons de proximité et non pas forcément de culture partagée. Ces résultats ne vont pas dans le sens d'un enfermement de type communautaire qui pourrait avoir un impact sur le développement.

Enfin, afin d'avoir une indication du niveau d'optimisme ou de pessimisme quant à l'avenir de leur pays, nous leur avons enfin demandé s'ils souhaiteraient dans le futur étudier ou vivre dans un autre pays que l'Algérie: 129 personnes ont répondu par l'affirmative, 45 par la négative et 8 n'ont pas répondu à cette question. Ces résultats dénotent un climat de pessimisme élevé par rapport à la situation de leur pays (cf. Tableau 6) qui est cohérent avec nos observations sur le terrain.

Tableau 6. Dans l'avenir souhaiteriez-vous étudier ou travailler dans un autre pays que l'Algérie?

\begin{tabular}{|l|l|l|l|l|l|l|}
\hline & \multicolumn{3}{l}{$\begin{array}{l}\text { Non } \\
\text { réponse }\end{array}$} & \multicolumn{3}{l|}{ Oui } \\
\hline & $n$ & $\%$ cit. & n & $\%$ cit. & n & $\%$ cit. \\
\hline Non réponse & 0 & 0 & 2 & 100 & 0 & 0 \\
\hline Étudiant(e) & 3 & 3,6 & 59 & 70,2 & 22 & 26,2 \\
\hline Sans emploi & 0 & 0 & 12 & 85,7 & 2 & 14,3 \\
\hline Agriculteurs exploitants & 0 & 0 & 0 & 0 & 0 & 0 \\
\hline Artisans, commerçants et chefs d'entreprise & 1 & 5,6 & 12 & 66,7 & 5 & 27,8 \\
\hline Cadres/prof. intellectuelles supérieures & 2 & 5,7 & 21 & 60 & 12 & 37,3 \\
\hline Professions intermédiaires & 0 & 0 & 7 & 70 & 3 & 30 \\
\hline Employés & 2 & 11,8 & 14 & 82,4 & 1 & 5,9 \\
\hline Ouvriers & 0 & 0 & 2 & 100 & 0 & 0 \\
\hline Total & & & 129 & & 45 & \\
\hline
\end{tabular}

\section{Conclusion}

La « valeur confiance » est-elle toujours aussi importante en Kabylie et sur quoi reposetelle dans ce territoire vécu (Perret et Gagnon 2016) et porteur d'une gouvernance villageoise qui puise ses racines dans la tradition?

La persistance d'une organisation sociale villageoise ancestrale (tajmaat) sous-tendue par un certain nombre de valeurs (tirugza), de pratiques (tiwizi), et qui est organisée autour de liens horizontaux (familiaux, tribaux, villageois) montre que le capital social est toujours important en Kabylie. Le lien social, qui est une ressource spécifique, devient alors un actif spécifique; la capacité à créer du lien devenant un capital. Dans les villages, la 
survivance de cette gouvernance ancestrale permet ainsi toujours un certain développement grâce à la participation des villageois aux projets collectifs financés par la caisse du village et réalisés grâce à leur travail bénévole (tiwizi). Cependant, la pérennité de cette valeur et, de fait, l'existence des comités de village sont aujourd'hui quelque peu « menacés » par l'amélioration du système redistributif et les migrations qui rendent les solidarités villageoises ou intergénérationnelles moins nécessaires à la survie.

Concernant la «vraie confiance » décrite par Laurent (2012), si la confiance envers les personnes appartenant aux groupes primaires est forte (notamment envers la famille avec une moyenne de 4,62 sur une échelle de 5 dans notre enquête exploratoire), celle envers les hommes politiques est faible ( 1,27 sur 5 pour les hommes politiques au niveau central). La confiance envers les institutions est également faible (1,53 sur 5 au niveau de L'État central et 1,59 sur 5 au niveau de la Wilaya), ce qui peut s'expliquer en partie par la perception de la corruption.

Concernant la confiance en l'avenir, qualifiée de «fausse confiance » par Laurent (2012), elle est particulièrement inquiétante puisqu'une grande majorité des personnes interrogées (129 sur 182) ont déclaré souhaiter étudier ou vivre un jour dans un autre pays que l'Algérie, et ce, qu'elles soient étudiantes ou en situation d'emploi.

Nous avons vu que l'appartenance à une subculture particulière pourrait donner des informations sur la façon dont on espère qu'un individu va se comporter (cf. la disposition pro-sociale de Dagupta (2010)). Par exemple, un Kabyle de tel ou tel village pourrait s'attendre à ce que celui qui appartient à la même subculture - qui repose sur un certain nombre de valeurs fortes (solidarité, entraide, etc.) - se comporte en argaz et lui faire confiance; la relation de confiance provenant ici du fait que les personnes concernées vivent dans une réalité commune au sein de laquelle elles peuvent s'attendre à des comportements réciproques. Cependant, il est évident que l'intégration dans un ensemble normatif intériorisé par les personnes d'une même communauté est une condition insuffisante à l'établissement d'une relation de confiance au sens vrai du terme (goodwill trust de Schuller, 2004, p. 9).

Pour revenir à la troisième partie de la question soulevée en introduction, à savoir l'existence ou non d'un renfermement communautaire nuisible au développement territorial, il ne semble pas y avoir de renfermement communautaire particulier qui pourrait nuire à la création d'entreprises et, par suite, à la dynamique territoriale. En effet, les personnes interrogées indiquent en majorité qu'en cas de création d'entreprise, il leur est indifférent que leur associé ne soit pas de la même wilaya de résidence $(59,4 \%$ de notre échantillon). Lorsqu'elles préfèrent un associé de leur wilaya, les raisons qu'elles avancent peuvent simplement relever d'une préoccupation de proximité géographique.

Enfin, si l'enquête exploratoire qualitative sur la confiance que nous avons menée ne permet pas de conduire toutes les opérations quantitatives qui se rattachent à une enquête statistique, elle permet cependant de concrétiser et documenter, de façon exploratoire, un sujet peu étudié en Kabylie. 


\section{BIBLIOGRAPHIE}

Adli Y., 2010-a, Les efforts de préservation de la pensée kabyle aux XVIII ${ }^{e}$ et XIX ${ }^{e}$ siècles, Tome 2, L'Odysée édition.

Adli Y., 2010-b, Les efforts de préservation de la pensée kabyle aux XVIII ${ }^{\mathrm{e}}$ et XIX ${ }^{\mathrm{e}}$ siècles, Tome 1 , L'Odysée édition.

Algan Y. et Cahuc P., 2007, La société de défiance. Comment le modèle social français s'autodétruit, éditions Rue d'Ulm.

Angeon V., Caron P. et Lardon S., 2006, « Des liens sociaux à la construction d'un développement territorial durable : quel rôle de la proximité dans ce processus? ", Développement durable et territoires, Dossier 7 Proximité et environnement, p. 1-21, http://

developpementdurable.revues.org/2851, consulté le 04/09/2014.

Arrow R., 1999, “Observation on social capital”, in Dasgupta P. et Serageldin I., 1999, Social capital. A multifaceted perspective, The World Bank, Washington.

Banque Mondiale, 2000, Combattre la pauvreté. Rapport sur le développement dans le monde, Washington.

Bourdieu P., 1958, réédition 2012, Sociologie de l'Algérie, PUF.

Bourdieu P., 1980, «Le capital social. Notes provisoires ", Actes de la recherche en sciences sociales, vol. 31, janvier, p. 2-3.

Bucolo E., 2004 , « Capital social, pour un regard critique », Actes du colloque Le capital social organisé par le Groupe de recherche Innovation et Sociétés, Université de Rouen 6 février 2003, Cellule GRIS n 10 , p. 69-80.

Burt R., 1995, « Le capital social, les trous structuraux et l'entrepreneur », Revue française de sociologie, XXXVI, p. 599-628.

Camps G., 2007, Les Berbères. Mémoire et identité, Actes Sud, Collection Babel, Arles.

Colman J. S., 1988, "Social capital in the creation of human capital", The American Journal of Sociology, 94, S95-S120.

Colletta, N. J., Cullen, M. L., 2000, The Nexus between Violent Conflict, Social Capital and Social Cohesion: Case Studies from Cambodia and Rwanda, World Bank, Washington DC.

Dasgupta P., 2010, « Une question de confiance : capital social et développement économique » Revue d'économie du développement, 2010/4, vol. 24, p. 47-96.

Hanoteau A., Letourneux A., 1893/2003, La Kabylie et les coutumes kabyles, seconde édition revue et argumentée, Tome deuxième, Éditions Bouchène.

Houard J. et Jacquemain M., 2006, Capital social et dynamique régionale Économie Société Régions, Collection de l'Institut wallon de l'évaluation, de la prospective et de la statistique (IWEPS), Éditions De Boeck Universités, Bruxelles.

Kamanzi C., Zhang X. Y., Deblois L. et Deniger M.-A., 2007, « L'influence du capital social sur la formation du capital humain chez les élèves résilients de milieux socioéconomiques défavorisés ", Revue des sciences de l'éducation, vol. 33, n 1, p. 127-145. 
Lacoste-Dujardin C., 2005, Dictionnaire de la culture berbère en Kabylie, La Découverte.

Laurent E., 2012, Économie de la confiance, Collection Repères, La Découverte.

Mercure D., Harricane B., Seghir S., Steenhaut A., 1997, Culture et gestion en Algérie, L'Harmattan.

Martens S., 2003, «Problématique de participation de la « société civile » aux stratégies de réduction de la pauvreté au Rwanda », in Marysse S. et Reyntjens F. (Direction), L'Afrique des grands lacs 2002-2003, p. 109-131.

Naves M.-C., 2003, « Démocratie, libéralisme et capital social. Une lecture de Trust de Francis Fukuyama ", Revue internationale de politique comparée, vol. 10, p. 477-488.

Pecqueur B., 2003, « Dans quelles conditions les objets patrimoniaux peuvent-ils être support d'activité ? ", XIII conférence internationale du RESER, Services et développement régional, Mons, 9 et 10 Octobre 2003, Ateliers de la Fucam.

Perret C., 2012, Territoire, identité et savoir-faire ancestraux. La poterie kabyle, collection Sciences Humaines Culture, Edilivre, Universitaire.

Perret C., Abrika B., 2014, « Les systèmes de gouvernance traditionnels en Kabylie à la lumière du concept de capital social » Mondes en développement, vol. 42-2014/2-n 166, p. 131-144.

Perret C., Gagnon C., 2016, « Identité(s), gouvernance villageoise et développement territorial viable en Kabylie », colloque du CIST - En quête de territoire(s) ?, Grenoble, 17\&18 mars 2016.

Putnam R., 1995, “Bowling alone: America's declining social capital”, Journal of Democracy, 6(1), p. 65-78.

Perret C., Paranque B., 2014, « Villages kabyles et gestion commune des ressources naturelles : entre modernité et tradition ", http://papers.ssrn.com/sol3/papers.cfm?abstract_id=2441204, consulté le 03/09/2014.

Schuller G., 2004, «Économie et confiance. La confiance, un facteur indispensable mais complexe », mimeo, janvier, 41 p. (http://www.portstnicolas.org/IMG/pdf/ ECO_et_confinace_Version07_2004.pdf, consulté le 20/02/2015).

Sirven N., 2003, « L'endogénéisation du rôle des institutions dans la croissance ou la (re)découverte du capital social », in Ballet et Guillon, Regards croisés sur le capital social, L'Harmattan, p. 57-90.

Solow R., 1999, "Notes on social capital and economic performance", in Dasgupta P. et Serageldin I., 1999, Social capital. A multifaceted perspective, The World Bank, Washington.

Woolcock, M., 1998, "Social Capital and economic development: Toward a theoretical synthesis and policy framework", in Theory and Society, vol. 27, p. 151-208.

Woolcock M., 2001, “The place of social capital in understanding social and economic outcomes", http://www.oecd.org/innovation/research/1824913.pdf, consulté le 04/09/2014.

Woolcock, M., Narayan, D., 2000, "Social capital: implications for development theory, research and policy", The World Bank Research Oberver, vol. 15, p. 225-249.

\section{NOTES}

1. Le taux d'homogénéité culturelle, qui compare le lieu de résidence et l'origine culturelle du père, montre que c'est l'aire des Monts de Kabylie qui est la plus homogène de toutes les subcultures algériennes (Mercure et al.,1997, p. 29). 
2. Bourdieu, 1958 réédité en 2012, p. 12.

3. Le territoire est le résultat d'un processus de construction et de délimitation par les acteurs. Il est le fruit d'une histoire et de traditions mais aussi de contraintes exogènes ou endogènes. Il est une réalité qui se cristallise dans différentes configurations et des modes de gouvernance particuliers. Il fait système mais il ne correspond pas forcément à des frontières administratives.

4. La caisse des villages kabyles est essentiellement alimentée par les cotisations des villageois (même émigrés), des dons et des recettes d'activités collectives diverses (fêtes, organisation de pèlerinages...).

5. Nous utilisons le terme organisation informelle dans le sens où ces comités, qui ne sont pas forcément déclarées sous la forme associative (c'est la tendance actuelle afin de disposer d'un compte bancaire pour la caisse du village) fonctionnent toujours en parallèle des institutions étatiques (Perret et Abrika, 2014).

6. Camps, 2007, p. 297.

7. Le règlement intérieur du village est rédigé en une série d'articles. Son contenu dépend des villages. Il prévoit le règlement de certains litiges et des sanctions financières en cas de non respect de la loi du village. Il ne se substitue cependant pas à la loi (Perret et Paranque, 2014).

8. Poète kabyle (1836-1901).

9. "La citoyenneté se réinvente en kabylie : Leçon de gouvernance à Iguersafène", El Watan, jeudi 12 Février 2015.

D'autres exemples de réalisations sont cités dans Perret et Abrika (2014).

10. Argaz (au pluriel irgazen) signifie Homme (adulte, en âge de porter les armes) chez les tous Berbères du Nord (Touaregs exclus). Le sens de la racine lexicale (RGZ), bien conservé en touareg, signifie précisément marcher en file indienne. Argaz, qui a une structure morphologique d'adjectif, est donc étymologiquement le marcheur, le piéton, le fantassin (échange avec Salem Chaker, Professeur de langue berbère).

11. L'Indice de Perception de la Corruption de Transparency International classe les pays en fonction du degré de corruption perçue dans les administrations publiques et la classe politique. 12. Un indice faible montre une très forte corruption.

13. En 1970, l'Italie avait créé des gouvernements locaux dans ses 20 régions et transféré certaines fonctions du gouvernement central (Thiébault, 2003, p. 342).

14. À partir de l'analyse des enquêtes du World Values Survey, Ronald Inglehart (1993) a constaté que «Parmi les pays pour lesquels nous avons des données de 1973 à nos jours, la France et l'Italie accusent les plus bas niveaux de satisfaction à l'égard de la vie, du bonheur, de la satisfaction politique et de la confiance" (Source : Algan T. et Cahuc P., mimeo, "Peut-on construire une société de confiance en France?" (http://econ.sciences-po.fr/sites/ default/files/file/yann\%20algan/Construire\%20une\%20societe\%20de\%20confiance_Albin \%20Michel.pdf).

15. Consultable sur le site: http://www.sphinxonline.net/Cecile/Confiancetraitement/ questionnaire.htm.

16. http://www.worldvaluessurvey.org/wvs/articles/folder_published/survey_1990/files/ root_q_1990.pdf.

17. Le quotidien Liberté.

18. Des comités d'autodéfense des villages avaient été constitués durant la décennie noire par la société civile. Ils ne sont plus actifs aujourd'hui. 


\section{RÉSUMÉS}

Des organisations informelles sont créées en l'absence d'institutions formelles fortes ou en cas de manque de confiance dans les institutions. Le capital social structurel civil, comme par exemple le système de gouvernance traditionnel des villages kabyles, peut alors se substituer au capital social structurel gouvernemental. Le mode d'organisation traditionnel kabyle, fondé sur un certain nombre de valeurs (démocratie, entraide...), privilégie les liens de type bonding qui caractérisent des relations communautaires. Mais, cet état de fait ne pourrait-il pas tendre vers un renfermement communautaire qui pourrait avoir un impact négatif sur la dynamique territoriale? Nous montrons, à l'aide d'une enquête exploratoire qualitative, que ce n'est pas le cas en Kabylie.

As numerous economists and anthropologists underline it, informal organizations are created in the absence of strong formal insitutions or in the case of lack of trustworthiness. Civil structural social capital, as the traditional villages' governance system in Kabylia, can then be substituted to the governmental structural social capital. This traditionnal organization, based on values such as mutual aid, equality, democracy... promotes the bonding ties wich characterize community relations. Can this situation engender a "relational closure" wich could have a negative impact on territorial dynamics? This research brings into light, with an original survey administrated in Tizi-Ouzou, that it's not the case.

\section{INDEX}

Mots-clés : lien social, capital social, confiance, gouvernance territoriale, développement territorial, Kabylie

Keywords : social ties, social capital, trustworthiness, territorial governance, territorial development, Kabylia

\section{AUTEURS}

\section{CÉCILE PERRET}

Cécile Perret est rattachée à l'IREGE (université de Savoie) et membre collaborateur au CRDT (université du Québec). Elle s'intéresse au rapport entre qualité des liens sociaux et développement des territoires, Cecile.perret@univ-smb.fr

\section{BELAÏD ABRIKA}

Belaïd Abrika est maître de conférences et dirige un master en économie à l'Université Mouloud Mammeri de Tizi-Ouzou. Il s'intéresse, notamment, à l'économie de la corruption, université Mouloud Mammeri, Tizi-Ouzou, belaidamazigh@yahoo.fr 